\title{
Research of the Ultrasonic Distance Measure
}

\author{
Min Jiao \\ Department of automotive, Sichuan Top IT Vocational Institute, Chengdu, 611743, China
}

Keywords: Ultrasonic Sensor; Distance Measure; Temperature Compensation; SCM.

\begin{abstract}
According to the problems of high-cost ultrasonic distance measure and liquid, solid surface is not easy to measure for the current measurement applications, a low-cost ultrasonic distance measure design scheme based on SCM was given. According to the characteristics of ultrasonic propagation in air, discussed the hardware and software design method of the ultrasonic distance measure, and temperature compensation method of upgrading measuring accuracy .Proved by the experiment, the scheme has the advantages of simple structure, reliable work and low cost, and has a certain practical value.
\end{abstract}

\section{Introduction}

Ultrasonic distance measure is a non-contact measurement method. At present, the ultrasonic ranging technology has been widely used in machinery manufacturing, metallurgy, electronic navigation, aerospace, petrochemical, transportation and other industries. Compared with other methods, such as method of electromagnetic or optical, which has no influence from light, colour of the measured object and has certain ability to adapt to the dark, dust, smoke, electromagnetic interference, toxic environment. So the application is widely used in the field of measurement, such as liquid level measurement, reversing radar, robot vision, but the ultrasonic distance measure is generally high cost sold on the market, the price is expensive. Aiming at the above problems, this paper presents the design of a low cost ultrasonic distance measure. Compared with the traditional distance measure, is simple in principle, easy to control, and has advantages of non-contact measure, low cost etc.

\section{The basic principles of ultrasonic distance measure}

In this paper, using pulse echo method for distance measure, the principle is the ultrasonic transmitter send out ultrasonic at a time, ultrasonic obstacle reflected back by the receiver receives the echo. Measured from the ultrasonic transmitting pulse time to receive the echo signal by the time $t$, then calculate the ultrasonic probe and the distance between the object to be measured is which is given by:

$$
s=(v \cdot t) / 2
$$

Among them, $\mathrm{s}$ is the object to be measured and the distance to the obstacle is the speed of sound in the air, $\mathrm{t}$ is the time when the sound waves bounce back and forth.

The ultrasonic is a kind of sound waves, the propagation velocity will be change with the ambient temperature change, in the actual situation need to correction by temperature compensation method. The relationship between the speed of sound and temperature in air can be expressed as:

$$
v \approx 331.45 \sqrt{\frac{T+273.16}{273.16}} \approx 331.4+0.6 T
$$

In the formula, $\mathrm{T}$ is the actual temperature $\left({ }^{\circ} \mathrm{C}\right)$.

Because it is the use of ultrasonic distance measurement to measure the expected distance, so the generated ultrasonic waves have a certain power and reasonable frequency can reach the predetermined transmission distance, and it is a necessary condition for the echo power enough to get, only to get echo frequency enough, receiving circuit can detect the echo signal and prevent interference from outside interference signal. Through analysis and experiments show that, the 
ultrasonic frequency is about $40 \mathrm{KHz}$ spread in the air the best effect, at the same time in order to facilitate treatment, the emitted ultrasonic wave is modulated into modulation with a certain interval of the pulse wave signal.

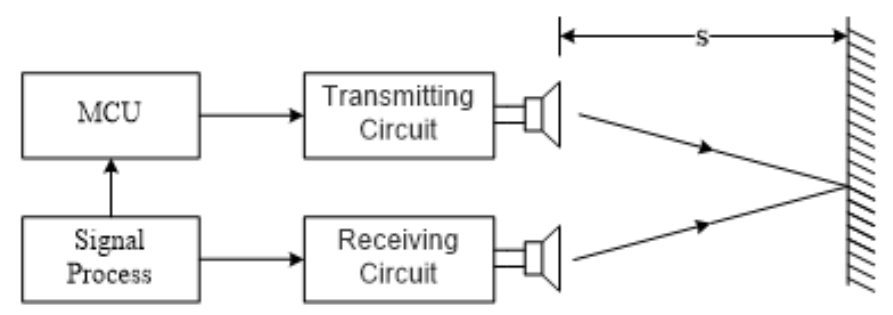

Fig.1: Ultrasonic distance measure basic circuit diagram

\section{The overall design of ultrasonic distance measure}

The basic thought of this system is based on the single chip microcomputer AT89C51, the ultrasonic sensor using the transceiver split, respectively is an ultrasound transducer TCT40-16T and an ultrasonic receiving transducer TCT40-16R.The ultrasonic signal emission to the air through the ultrasound transducer, after being reflected by the object, echo is received by ultrasonic transducer. The whole system consists of ultrasonic transmitting / receiving circuit, display circuit, temperature detecting circuit, control circuit, alarm circuit. Ultrasonic transmitting /receiving circuit mainly realizes sending and receiving ultrasonic; temperature detection circuit mainly measuring environment temperature, realize the temperature compensation. The display circuit to realize the measurement data display, MCU as the control circuit, receiving data to complete the instruction sending, and calculate task. The system structure diagram as shown in figure 2.

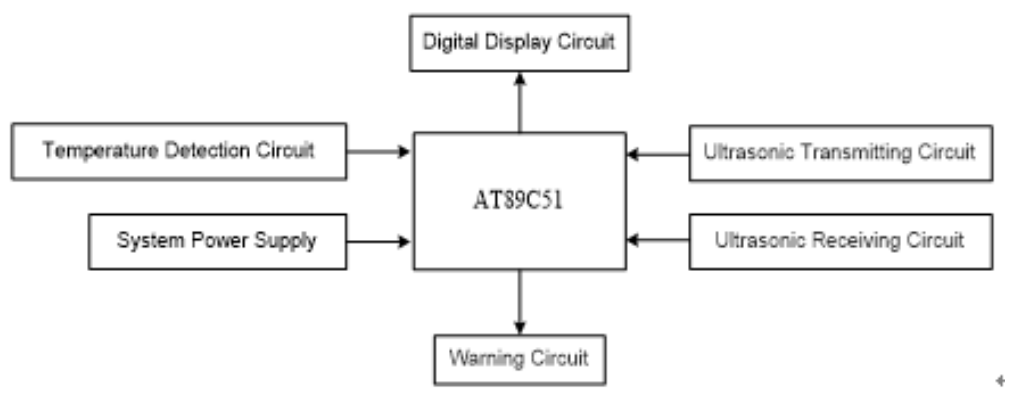

Fig. 2: Ultrasonic distance measure system structure diagram

\section{System hardware design}

\section{Ultrasonic transmitting circuit}

The transmitting circuit output of about $40 \mathrm{KHz}$ square wave pulse signal by AT89C51 P1.0 port,which also open internal counter T0 when the first signal is issued. As the SCM port output power is very weak, in order to make the distance measure meet the requirements, to drive the ultrasonic sensor of ultrasonic transmitting distance far enough.Therefore, need to increase the power on circuit.

Pulse signal transmitting circuit used to provide enough power for the ultrasonic transmitter is completed by the CD4069 inverter.The output of the $40 \mathrm{kHz}$ square wave signal is send to one electrode of the ultrasonic transducer via one-stage reverser,the other path another electrode via two-stage reverser is sent to the ultrasonic transducer both ends of the square wave signal, will be added to the ultrasonic transducer with the push-pull form. In this way, the transmitter can get the peak to peak value of $9 \mathrm{~V}$ AC sine wave (ultrasonic transmitter $\mathrm{Q}$ value is very high, only the fundamental signal effect, no influence of higher harmonics) signal, thus improves the transmitting power.To meet the requirements of measuring distance, finally gave the ultrasound transducer 
TCT40-16T, in the form of sound wave emitted into the air. The transmitting part of the circuit, as shown in figure 3.

\section{Ultrasonic receiving circuit}

The main task of receiving circuit is to detect the echo signal that is the ultrasonic receiving transducer.

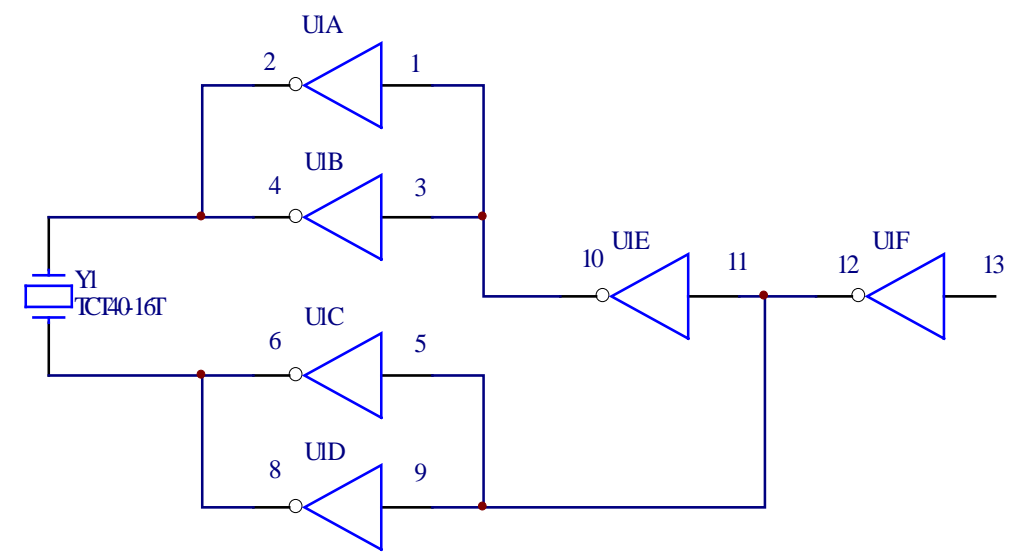

Fig. 3: ultrasonic transmitting circuit principle diagram

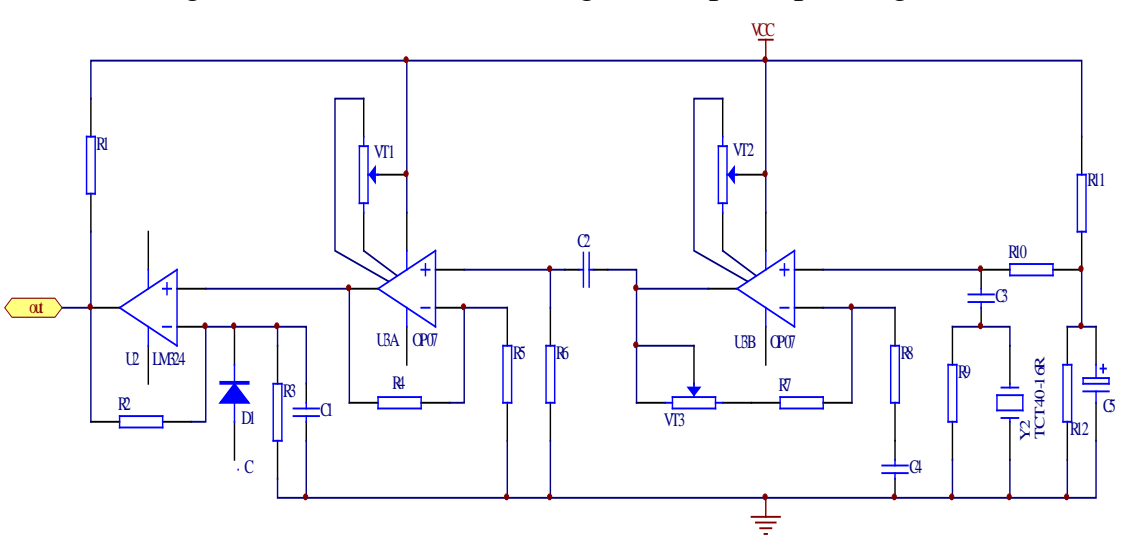

Fig. 4: ultrasonic receiving circuit principle diagram

TCT40-16R successfully received echo, converted into electrical signal, and after the signal being amplified, filtering, shaping treatment, then send an interrupt signal to stop the timer to MCU. The design of receiving circuit will have a direct impact on the measurement of the propagation time of ultrasonic wave in the air. Ultrasonic receiving circuit mainly includes the weak signal amplifier, voltage compare interrupt signal output. Because the ultrasonic echo signal is very weak and noisy, $\mathrm{S} / \mathrm{N}$ is smaller, so the receiving circuit is provided with two levels of high $\mathrm{Q}$ value of the filter amplifier circuit.

The system uses two-stage amplifying circuit composed of two pieces of OP07, to amplify the received ultrasonic signal. Stage one and stage two filter amplifier circuit using the same structure and parameters. After the signal is amplified, input to the positive terminal of LM324, compared with the reference voltage. When the output is low, there are two possibilities: no echo signal or the echo signal is very weak, beyond the scope. The system identified both as no echo signal; otherwise output high level, the system identified as the echo signal, immediately stop timer.The receiveing part of the circuit, as shown in figure 4.

\section{Temperature detection circuit}

The main task of temperature detection circuit is to get real-time measurement ambient temperature, so that the system can accurately calculate the actual ultrasonic wave velocity, so as to improve the precision of the measuring system. The temperature measurement of this system using DS18B20 digital thermometer produced by DALLAS.

The DS18B20 communicates over a 1wire bus that by definition requires only one data line for communication with SCM, a great convenience to users to debug. And, is accurate to $\pm 0.5^{\circ} \mathrm{C}$ over the range of $-10^{\circ} \mathrm{C}$ to $+85^{\circ} \mathrm{C}$. The data signal is obtained by DS18B20 via a 
single bus interface to SCM, through calculating to get the real-time environmental temperature value.

\section{System software design}

The design procedure uses the modular programming, including the main program module, ultrasonic ranging program module, display module, temperature measurement module and external interrupt module. The ultrasonic ranging work process is as follows: pulse signal is generated by AT89C51.Each measurement need to emit at least 10 complete $40 \mathrm{KHz}$ pulse. At the same time open internal counter T0 when the first signal is issued. When the counter reaches a certain value and then opening detecting echo signal, in order to avoid the interference of the aftermath. Using external interrupt to detect the echo signal. Once received echo signal, immediately read the value in the counter, this data is the time need to be measured .In order to avoid the error of measured data, processing method for range data in the program is: for every location, need to measure repeatedly, can obtain multiple sets of data, these data will be processed to obtain the distance value. The detail flow chart as shown in figure 5 .

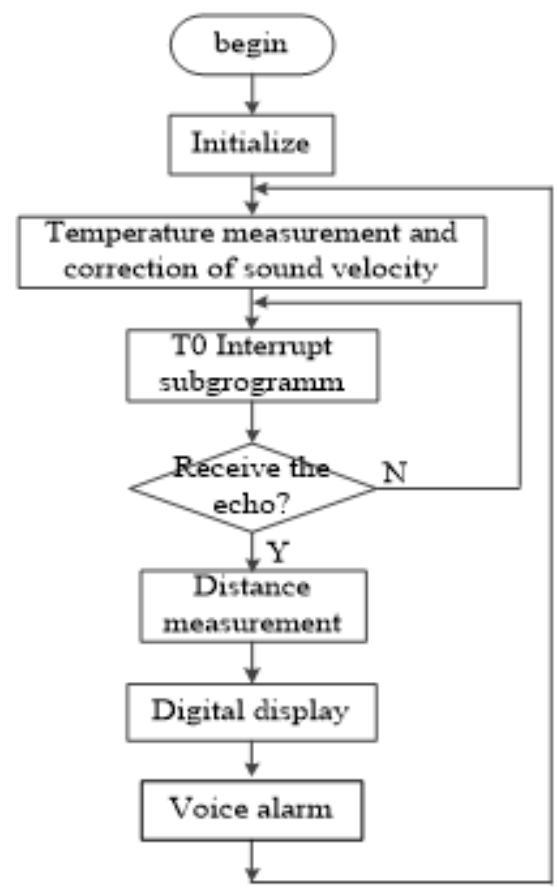

Fig. 5: Ultrasonic distance measure design flow chart

\section{Conclusion}

This paper mainly discusses to realize the non-contact distance measurement using ultrasonic sensor, and fully considers the influence of environment temperature on the measuring ultrasonic velocity, through the method of temperature compensation for sound velocity corrected, so it has high measurement precision. Because this system has the characteristics of simple structure, reliable work and low cost, and the recognition of vehicle automatic navigation and other fields can be applied to all kinds of liquid level measurement, obstacle, it has broad application prospects.

\section{References}

[1] Linna Wang, Cuili Luo, Hui Ma; Circuit design of ultrasonic range finder [J]; Application of power technology; 201302

[2] Zongzhou Wen, Funing Li, Zibang Xia; Design of high precision ultrasonic distance measurement system [J]; Instrument technique and sensor; 201211 
[3] Xiaoqiang Zhao; simplified ultrasonic telemeter system [J]; Journal of Tianjin University of Technology; 201001

[4] Min Zhu; ultrasonic ranging error and the correction method of the analysis [J]; measurement and testing technology; 200902

[5] Fengying Niu, Hua Zhang, Jizhong Liu; Wuchang Su; intelligent wheelchair obstacle avoidance control system [J]; sensor and micro system; 200810

[6] Shunxian Bai; Design and implementation of the ultrasonic ranging system [J]. Information of science and Technology (Research), 2007 (27)

[7] Lifang Xue, Hui Wang, Wenjun Yan; Ultrasonic distance measurement based on [J].automation and instrumentation, 200705

[8] Wei Shi, Jun Meng, Liu Paul; The design of temperature control for ultrasonic ranging correction [J]; mechanical engineering and automation; 200506. 